\title{
Renewed Focus on the Threat of Cross-Contamination in Molecular Laboratories: Notes from the COVID-19 Testing Experience in Bangladesh
}

\author{
Mohammad Jahidur Rahman Khan 1*, Ruksana Raihan², S. M. Rashed Ul Islam³, \\ Nusrat Mannan', Mohammad Jamal Uddin", Nazia Hasan Khan5, Tanzila Rawnuck6, \\ Amirul Huda Bhuiyan7, Farzana Mim8, Md. Selim Reza9 \\ ${ }^{1}$ Department of Microbiology, Shaheed Suhrawardy Medical College, Dhaka, Bangladesh \\ ${ }^{2}$ Department of Microbiology, US-Bangla Medical College, Dhaka, Bangladesh \\ ${ }^{3}$ Department of Virology Bangabandhu Sheikh Mujib Medical University, Dhaka, Bangladesh \\ ${ }^{4}$ Department of Microbiology \& Mycology, National Institute of Preventive and Social Medicine, Dhaka, Bangladesh \\ ${ }^{5}$ RT-PCR LAB, United Hospital Limited, Dhaka, Bangladesh \\ ${ }^{6}$ Department of Pathology \& Microbiology, Dhaka Dental College, Dhaka, Bangladesh \\ ${ }^{7}$ Department of Virology, Dhaka Medical College, Dhaka, Bangladesh \\ ${ }^{8}$ Department of Biochemistry \& Molecular Biology, Jahangirnagar University, Dhaka, Bangladesh \\ ${ }^{9}$ RT-PCR LAB, Bangabandhu Sheikh Mujib Medical College, Faridpur, Bangladesh \\ Email: *jahid29th@gmail.com
}

How to cite this paper: Khan, M.J.R., Raihan, R., Islam, S.M.R.U., Mannan, N., Uddin, M.J., Khan, N.H., Rawnuck, T., Bhuiyan, A.H., Mim, F. and Reza, Md.S. (2021) Renewed Focus on the Threat of Cross-Contamination in Molecular Laboratories: Notes from the COVID-19 Testing Experience in Bangladesh. Advances in Infectious Diseases, 11, 357-365.

https://doi.org/10.4236/aid.2021.114032

Received: September 26, 2021

Accepted: October 31, 2021

Published: November 3, 2021

\begin{abstract}
Rapid and accurate laboratory diagnosis of SARS-CoV-2 infection is crucial for the management of COVID-19 patients and control of the spread of the virus. At the start of the COVID-19 pandemic, Bangladesh had only one government molecular laboratory where real-time RT-PCR would be performed to diagnose SARS-CoV-2 infection. With the increasing number of suspected cases requiring confirmation diagnostic testing, there is a requirement to expand capacity for large-scale testing quickly. The government of Bangladesh established over 100 molecular laboratories within one year to test COVID-19. To expand the testing capacity, the government was compelled to recruit laboratory staff with limited experience and technical expertise, especially in molecular assays, to process specimens, interpret results, troubleshoot. As a result, the risk of diagnostic errors, such as cross-contamination, increased, potentially undermining the efficacy of public health policies, public health response, surveillance programs, and restrictive measures aimed toward containing the outbreak. In this piece, we discuss the different sources of crosscontamination in the COVID-19 RT-PCR laboratories and proffer practical
\end{abstract}


Copyright (C) 2021 by author(s) and Scientific Research Publishing Inc. This work is licensed under the Creative Commons Attribution International License (CC BY 4.0).

http://creativecommons.org/licenses/by/4.0/ preventive measures to avoid them.

\section{Keywords}

SARS-CoV-2, RT-PCR, Cross-Contaminations, Quality Control, COVID-19, False Positive

\section{Introduction}

The coronavirus disease 2019 (COVID-19) caused by SARS-CoV-2 has triggered a global public health emergency. Due to the rapid spread of SARS-CoV-2 infection, there were widespread shortages of personal protective equipment (PPE), diagnostic test kits, and vital patient treatment equipment. The laboratory diagnosis of SARS-CoV-2 infection is an essential aspect of resolving the present pandemic [1]. For detecting SARS-CoV-2 nucleic acids, Reverse transcriptase-polymerase chain reaction (RT-PCR) is the gold standard for laboratory diagnosis. Testing is essential to identify infected people and track down their contacts [2]. In Bangladesh, the first confirmed case of COVID-19 was announced on March 8, 2020 [3]. At the beginning of the pandemic, like many other countries, Bangladesh also had limited testing facilities to diagnose SARS-CoV-2 infection. Bangladesh boosted its testing capability by setting up more than one hundred COVID-19 dedicated RT-PCR laboratories within a year. As of September 7, 2021, 56 government and 83 private COVID-19 dedicated RT-PCR laboratories are running across the country [3]. The government of Bangladesh made a substantial investment in the public health molecular laboratories under the Ministry of Health and Family Welfare. This included the set-up of the necessary instruments and test kits and personnel recruitment. This article will focus on the risk of cross-contamination in the testing laboratories in Bangladesh, a country like other low-income countries, which had to quickly ramp up capacity with limited resources [4]. With these notes from the field, we discuss the various sources of contamination in COVID-19 RT-PCR laboratories and provide efficient, effective, and feasible solutions to address these issues.

\section{Methodology}

For this study, we conducted a literature review of the risk of diagnostic errors, such as cross-contamination to the laboratory diagnosis of COVID-19. We searched for articles published in English on the WHO website, peer-reviewed articles on Google Scholar and PubMed, official public health the government of Bangladesh. We used the following keywords: RT-PCR, cross-contaminations, quality control, COVID-19, false positive.

\section{Preanalytical Considerations}

Different types of errors can occur in molecular laboratories before, during, and after RT-PCR result analysis which may lead to cross-contamination (Table 1). 
The most crucial preanalytical error is the sampling step and selecting proper sampling locations including nose and throat, tracheal tube, and sputum. Standard equipment such as synthetic swabs made of dacron or nylon with aluminum or plastic shaft should be used for sampling. Nasopharyngeal (NP) and oropharyngeal (OP) swabs are the recommended specimens for diagnosing SARS-CoV-2 infection by RT-PCR. Sputum, endotracheal aspirate, or bronchoalveolar lavage can be used in the case of ambulatory patients or patients with more severe lung disease or lung tissue post-mortem [5]. Combined NP/OP swabs may increase the positivity rate [6]. Each sample should be appropriately identified and sufficient for testing [7]. Many countries are constrained by a lack of PPEs and a scarcity of human resources, while the number of suspected cases requiring confirmation testing increases exponentially [8]. With limited resources and an overburdening workload, adhering to the recommended protocols may be difficult. Still, it should not be overlooked because breaking them can result in immediate cross-contamination, jeopardizing the accuracy and quality of RT-PCR testing while resulting in an increased risk of laboratory-acquired infections [9].

All PPEs (disposable gown, gloves, cap, shoe cover, protective eyewear, and an N95 respirator mask) must be sterilized and worn in the correct order before specimen collection. When using gloves, make sure they cover a portion of the forearm while remaining under the sleeves to avoid skin exposure. To cover up the sleeves, a second pair of gloves might be used. PPEs must be worn at all times, including the gown, FFP2 (N95), goggles or face shield, and gloves [10]. Shaving is also recommended for male health workers to ensure that the mask adheres to their faces correctly [11]. The patient must be comfortable with their head resting against a plexiglass partition when the sample is taken. The swab should be placed to a suitable transport medium after sampling. Viral transport media (VTM), isotonic saline solution, tissue culture solution, and phosphate

Table 1. Possible sources of cross-contamination in a COVID-19 molecular testing laboratory.

\begin{tabular}{ll}
\hline & - Incorrect sampling method and sampling location \\
& - Inappropriate material for sampling \\
Pre-analytical error & - Inadequate sample volume \\
& - Inadequate or improper use of PPE \\
& - Lack of human resources \\
& - Low quantity and quality of extracted RNA \\
& - RNA contamination during extraction \\
& - Pipetting errors \\
& - Use of inappropriate sample volume for cDNA synthesis and \\
& - Improper storage of PCR reagents \\
Analytical error & - Misinterpretation \\
\hline Post-analytical error & - Incorrect determination of exact baseline and threshold \\
& - Amplicon contamination
\end{tabular}


buffer can be used as proper media for sample transportation. The tubes are then correctly labeled with the patient's personal information [12] [13]. Samples should be tightly capped and transported to the corresponding laboratory in biohazard zip-lock bags within a leak-proof icebox. The biohazard label outside the box should be visible [14]. Proper labeling, handling, and storage of obtained samples are necessary to avoid false-positive and false-negative results [15].

To avoid cross-contamination, it's necessary to change gloves and clean the workspace between each collection. Suppose it is impossible or practical due to a lack of resources and people on top of a heavy workload. In that case, another option is to disinfect gloved hands with $70 \%$ alcohol in a squeeze or spray bottle and then dry with fresh paper towels after each patient. Surfaces of the collecting booth, whether made of plastic or metal or covered with a nonporous cover, should be disinfected as well, especially if patients have come into personal touch with the area. Disinfectants such as sodium hypochlorite or bleach (0.1 percent for general surface disinfection and $1 \%$ for sample spill disinfection), 62 - 71 percent ethanol, 0.5 percent hydrogen peroxide, quaternary ammonium compounds, and phenolic compounds (used according to manufacturer's recommendations) have all been recommended by the WHO. Benzalkonium chloride or 0.02 percent chlorhexidine digluconate can also be utilized, albeit they are less effective. Aside from choosing the proper disinfectant, the contact time, dilution, and shelf life should be considered. Alcohol can also be sprayed, but only after at least 20 seconds of contact with the surface should it be cleaned. A new solution has to be made each time when using bleach [16]. Regular disinfection is also required for sample collecting boxes or coolers, reusable cold packs, pouches, and racks. However, after disinfection, the technician must clean the surfaces with a sterile water-soaked paper towel followed by a 70 percent alcohol-soaked paper towel to avoid residue build-up and PCR inhibition.

When sampling is completed, PPEs should be removed properly to avoid contact with exterior surfaces. The used suit, shoes, gloves, and mask must all be disposed of in a specific garbage receptacle. Hands are also sanitized with an alcoholic solution or washed with soap and water [11]. Due to limited resources, laboratory staff is forced to operate under extreme pressure in high-throughput environments with an overwhelming workload and inadequate access to personal protective equipment as the number of suspected cases requiring confirmatory diagnostic testing grows [17]. To meet this demand, the laboratories had to recruit additional laboratory personnel with limited experience, and technical knowledge, in molecular assays. As a result, laboratory medical services become more vulnerable to diagnostic errors, including cross-contamination. They have a higher risk of producing false-positive results, which can jeopardize the patient's health and undermine the efficacy of public health policies, public health response, surveillance programs, and restrictive measures for containing the outbreak [18]. In the worst-case scenario, a false-positive result may result in wasteful treatment. It may jeopardize the available workforce, mainly if the pa- 
tient is a public servant obliged to self-isolate. Due to failures in concerning restraining and containment measures and identifying other suspected cases, particularly those exposed to the patient infected with SARS-CoV-2, a false-negative result can foster the rapid human-to-human transmission of the virus [19].

\section{Analytical Concerns}

When detecting unique sequences of the SARS-CoV-2 genome, RT-PCR remains the gold standard [20]. RT-PCR is a labor-intensive and intrinsically complex assay requiring extensive testing knowledge in all aspects, limiting the potential for rapid turnaround time from sample collection to data availability. This bottleneck could result in extended wait times and an exponential increase in testing demand [7].

The World Health Organization released guidelines on biosafety in laboratories handling COVID-19 samples [21]. The directorate general of health services of Bangladesh also released guidelines on how to operate local COVID-19 testing laboratories [3]. These initiatives establish a standard for ensuring the accuracy of tests and ensuring the safety of laboratory personnel. RT-PCR diagnostic kits have a high rate of false-negative test results. Unnecessary mistakes have to be prevented during the collection and processing of samples [22]. All samples should be processed within a class 2 biological safety cabinet (BSC) in full PPE attire as described above [21].

Due to the complexity of the RT-PCR test procedure, it is vulnerable to cross-contamination. For several testing kits, RNA from the COVID-19 suspected sample needs to be extracted.

The vital part of the analytical error is the Nucleic acid extraction step. If we don't get pure RNA, then it will give a false positive or false-negative result. To avoid this, we should choose a proper RNA extraction procedure for a valid real-time PCR result. SARS-CoV-2 RNA can easily be transferred from an infected gloved hand to a working surface or a laboratory environment [23]. Although WHO guidelines recommend excellent microbiological techniques and procedures, it is unclear how frequently laboratory employees should replace gloves. The rules also place a greater emphasis on safeguarding laboratory staff. To avoid cross-contamination, gloves should be changed as often as possible, especially if they have been soiled with solutions containing template RNA. Materials including pens, tiny equipment, tubes, pipette tips, and other consumables should never be brought from the RT-PCR area to the pre-PCR area. Laboratorians and even cleaning staff should be reminded that laboratory standards necessitate unidirectional workflow. Thus, they should regard each space as a separate room to avoid transporting amplicons to amplification product-free regions. Furthermore, according to the DGHS guideline of Bangladesh, the pre-PCR room must be divided into specimen handling or sample preparation room and reagent preparation room. Positive internal reaction controls are prohibited in the reagent preparation area, which should remain a "template-free" environment. The samples and reagents should be stored in separate 
freezers [3].

Pipetting patient samples into the PCR plate or strip is another probable cause of cross-contamination. Due to sample misplacement, negative samples can be mistaken as positive [24]. When doing RT-PCR analysis, correct pipetting and double-checking sample placement should always be followed while following aseptic practices (use of PPE, use of sterile materials, sanitizing work environment). Before and after PCR operations, cleaning the work environment, pipettors, freezer handles, and other equipment with the necessary decontaminating solution is also required. Racks should be disinfected for ten minutes before being dried with a clean paper towel. Autoclavable pipettors should be used to prevent cross-contamination. Disinfectants should be used as recommended by the WHO [21], either every 30 minutes or following COVID-19 sample processing [16]. Disposables are indicated for consumables that have come into touch with infectious material.

\section{Post Analytical Considerations}

Following RT-PCR analysis, post-PCR is a critical step in diagnosing the data. To ensure that the process is free of contamination, no amplification must be detected in the negative controls provided by the test kit, as well as in the elution buffer (or whatever is appropriate depending on the test kit used). In the event of possible contamination, the quality of the water should be verified, and contamination of the instrument should be considered in some circumstances [25]. To avoid these issues, each run should utilize new (unopened) water, and once the kit is opened, the reagents should be prepared in aliquots in sterile containers. Until the samples are deposited in the machine, the proper aseptic method must be followed. It is recommended that the controls not be placed adjacent to each other when inserting samples and controls in the multi-well plate to avoid cross-contamination [26]. In contrast, samples are transported to their allotted wells. To monitor aseptic pipetting, assigning about three or more water controls at random in the multi-well plate is also a good idea. Laboratory cross-contamination isn't always the cause of false data. Contamination in the test kits caused a delay in testing in Europe during the early stages of the COVID-19 pandemic [27].

Technicians working in a COVID-19 testing facility may become infected with the virus and unintentionally contaminate the samples they analyze and the laboratory environment. As a result, technicians, particularly those assigned to the PCR room, should wear goggles or a face shield and a disposable surgical hat and mask, which must be disposed of in designated receptacles in the same room before departing.

\section{Conclusion}

Laboratory diagnosis of SARS-CoV-2 infection by RT-PCR method plays one of the most crucial parts of the management of COVID-19 patients and controls 
the spread of the virus. Despite remarkable progress in the scale-up of testing capacity in the country, the testing facilities for COVID-19 disease are still not sufficient in Bangladesh. The guidelines published by the World Health Organization and the directorate general of health services should be strictly followed across all stages of testing. Particular attention should be given to avoid any cross-contamination during sample collection from suspected COVID-19 cases, changing gloves as often as possible and changing PPE when moving from one working place to another within the laboratory. Practicing unidirectional workflow and following aseptic technique in every step is vital in maintaining the quality of testing of a molecular diagnostic laboratory.

\section{Conflicts of Interest}

The authors declare no conflicts of interest regarding the publication of this paper.

\section{References}

[1] Araz, O.M., Ramirez-Nafarrate, A., Jehn, M. and Wilson, F.A. (2020) The Importance of Widespread Testing for COVID-19 Pandemic: Systems Thinking for Drive-Through Testing Sites. Health Systems, 9, 119-123. https://doi.org/10.1080/20476965.2020.1758000

[2] Layfield, L.J., Camp, S., Bowers, K. and Miller, D.C. (2021) SARS-CoV-2 Detection by Reverse Transcriptase-Polymerase Chain Reaction Testing: Analysis of False-Positive Results and Recommendations for Quality Control Measures. Pathology-Research and Practice, 225, Article ID: 153579. https://doi.org/10.1016/j.prp.2021.153579

[3] Hossain, I., Mullick, A.R., Khan, M.H., Ahmad, S.A., Rahman, M.S. and Aktaruzzaman, M.M. (2020) Epidemiology of Coronavirus Disease: Past, Present, Future Prospects and Its Journey towards Bangladesh. International Medical Journal, 25, 2517-2529.

[4] Manzano, J.A. et al. (2021) Repurposing Multi-Targeting Plant Natural Product Scaffolds in Silico against SARS-CoV-2 Non-Structural Proteins Implicated in Viral Pathogenesis. Cambridge Open Engage, Cambridge.

[5] Venter, M. and Richter, K. (2020) Towards Effective Diagnostic Assays for COVID-19: A Review. Journal of Clinical Pathology, 73, 370-377. https://doi.org/10.1136/jclinpath-2020-206685

[6] Wang, W., et al. (2020) Detection of SARS-CoV-2 in Different Types of Clinical Specimens. JAMA, 323, 1843-1844. https://doi.org/10.1001/jama.2020.3786

[7] Lippi, G., Simundic, A.M. and Plebani, M. (2020). Potential Preanalytical and Analytical Vulnerabilities in the Laboratory Diagnosis of Coronavirus Disease 2019 (COVID-19). Clinical Chemistry and Laboratory Medicine (CCLM), 58, 1070-1076. https://doi.org/10.1515/cclm-2020-0285

[8] Nemat, A., et al. (2021) A Survey of the Healthcare Workers in Afghanistan during the COVID-19 Pandemic. American Journal of Tropical Medicine and Hygiene, 104, 537-539. https://doi.org/10.4269/ajtmh.20-1367

[9] Kaufer, A.M., Theis, T., Lau, K.A., Gray, J.L. and Rawlinson, W.D. (2020) Laboratory Biosafety Measures Involving SARS-CoV-2 and the Classification as a Risk Group 3 Biological Agent. Pathology, 52, 790-795.

https://doi.org/10.1016/j.pathol.2020.09.006 
[10] Hase, R., Kurita, T., Muranaka, E., Sasazawa, H., Mito, H. and Yano, Y. (2020) A Case of Imported COVID-19 Diagnosed by PCR-Positive Lower Respiratory Specimen but with PCR-Negative Throat Swabs. Infectious Diseases, 52, 423-426. https://doi.org/10.1080/23744235.2020.1744711

[11] Piras, A., et al. (2020) Nasopharyngeal Swab Collection in the Suspicion of Covid-19. American Journal of Otolaryngology, 41, Article ID: 102551. https://doi.org/10.1016/j.amjoto.2020.102551

[12] Irving, S.A., Vandermause, M.F., Shay, D.K. and Belongia, E.A. (2012) Comparison of Nasal and Nasopharyngeal Swabs for Influenza Detection in Adults. Clinical Medicine \& Research, 10, 215-218. https://doi.org/10.3121/cmr.2012.1084

[13] Spencer, S., et al. (2013) Consistency of Influenza A Virus Detection Test Results across Respiratory Specimen Collection Methods Using Real-Time Reverse Transcription-PCR. Journal of Clinical Microbiology, 51, 3880-3882.

[14] Tan, S.S., et al. (2021) Practical Laboratory Considerations Amidst the COVID-19 Outbreak: Early Experience from Singapore. Journal of Clinical Pathology, 74, 257-260. https://doi.org/10.1136/jclinpath-2020-206563

[15] Loeffelholz, M.J. and Tang, Y.W. (2020) Laboratory Diagnosis of Emerging Human Coronavirus Infections-The State of the Art. Emerging Microbes \& Infections, 9, 747-756. https://doi.org/10.1080/22221751.2020.1745095

[16] Loh, T.P., et al. (2020) Operational Considerations and Challenges of Biochemistry Laboratories during the COVID-19 Outbreak: An IFCC Global Survey. Clinical Chemistry and Laboratory Medicine, 58, 1441-1449. https://doi.org/10.1515/cclm-2020-0710

[17] Sheridan, C. (2020) Coronavirus and the Race to Distribute Reliable Diagnostics. Nature Biotechnology, 38, 382-384. https://doi.org/10.1038/d41587-020-00002-2

[18] Giri, A.K. and Rana, D.R. (2020) Charting the Challenges behind the Testing of COVID-19 in Developing Countries: Nepal as a Case Study. Biosafety and Health, 2, 53-56. https://doi.org/10.1016/j.bsheal.2020.05.002

[19] Munne, K., Bhanothu, V., Bhor, V., Patel, V., Mahale, S.D. and Pande, S. (2021) Detection of SARS-CoV-2 Infection by RT-PCR Test: Factors Influencing Interpretation of Results. VirusDisease, 32, 187-189. https://doi.org/10.1007/s13337-021-00692-5

[20] Zheng, L., et al. (2020) Analysis of the Infection Status of Healthcare Workers in Wuhan during the COVID-19 Outbreak: A Cross-Sectional Study. Clinical Infectious Diseases, 71, 2109-2113. https://doi.org/10.1093/cid/ciaa588

[21] WHO (2020) Laboratory Biosafety Guidance Related to Coronavirus Disease (COVID-19): Interim Guidance, May 13 2020. World Health Organization.

[22] Younes, N., et al. (2020) Challenges in Laboratory Diagnosis of the Novel Coronavirus SARS-CoV-2. Viruses, 12, Article ID: 582. https://doi.org/10.3390/v12060582

[23] Lv, J., Yang, J., Xue, J., Zhu, P., Liu, L. and Li, S. (2020) Detection of SARS-CoV-2 RNA Residue on Object Surfaces in Nucleic Acid Testing Laboratory Using Droplet Digital PCR. Science of The Total Environment, 742, Article ID: 140370. https://doi.org/10.1016/j.scitotenv.2020.140370

[24] Albano, P.M., Notarte, K.I., Macaranas, I. and Maralit, B. (2020) Cross-Contamination in Molecular Diagnostic Laboratories in Low-and Middle-Income Countries. Philippine Journal of Pathology, 5, $5 \mathrm{p}$.

[25] Van Zyl, G., Maritz, J., Newman, H. and Preiser, W. (2019) Lessons in Diagnostic Virology: Expected and Unexpected Sources of Error. Reviews in Medical Virology, 
29, e2052. https://doi.org/10.1002/rmv.2052

[26] Barreto, H.G., de Pádua Milagres, F.A., de Araújo, G.C., Daúde, M.M. and Benedito, V.A. (2020) Diagnosing the Novel SARS-CoV-2 by Quantitative RT-PCR: Variations and Opportunities. Journal of Molecular Medicine, 98, 1727-1736. https://doi.org/10.1007/s00109-020-01992-x

[27] Mögling, R., et al. (2020) Delayed Laboratory Response to COVID-19 Caused by Molecular Diagnostic Contamination. Emerging Infectious Diseases, 26, 1944-1946. https://doi.org/10.3201/eid2608.201843 